\title{
Complejidad y cuidado emergente
}

\author{
Cecilia Ré *
}

\section{Resumen}

La postmodernidad surge con el paradigma de las Ciencias de la Complejidad (CC) con apoyo a teorías, modelos y prácticas de cuidado holístico. Rompen con postulados prevalentes. Abiertas al desarrollo teórico innovador, la experimentación y la investigación, reconstruyen los conocimientos con omnipresencia cognoscitiva universalista. Fundadas en la física cuántica, las matemáticas cualitativas y la informática, los conocimientos y las teorías científicas galenas están en revisión, aunque cierras los ojos ante ciertos hallazgos. La Enfermería tiene la oportunidad de superar los límites lineales impuestos por la estructura racional científica, evidente, circunscripta a la materialidad, acobijadas por el modelo médico hegemónico ( $\mathrm{MMH})$.

Palabras clave: $\quad$ Enfermería - Currículo - Holismo - Terapias alternativas Beneficios - Políticas 


\title{
Complexity and emergent care
}

\author{
Cecilia Ré *
}

\begin{abstract}
Postmodernity arises with the paradigm of Complexity Sciences (CC) with support for theories, models, and practices of holistic care. They break with prevalent postulates. Open to innovative theoretical development, experimentation, and research, they reconstruct knowledge with universalistic cognitive omnipresence. Founded on quantum physics, qualitative mathematics and computer science, Galena's scientific knowledge and theories are under review, although you close your eyes to certain findings. Nursing has the opportunity to overcome the linear limits imposed by the evident scientific rational structure, circumscribed to materiality, sheltered by the hegemonic medical model (MMH).
\end{abstract}

Keywords: Nursing - Curriculum - Holism - Alternative therapies - Benefits Policies 


\section{Introducción}

"Los doctores son hombres que prescriben medicinas que conocen poco, curan enfermedades que conocen menos, en seres de humanos de los que no saben nada". Voltaire (1694-1778)

Occidente funda el conocimiento científico separando las realidades universales. Promulgado por Isaac Newton, René Descartes, Francis Bacon, Augusto Comte, aniquila el integrismo presente en filosofías indias, taoístas, ancestrales australianas y autóctonas latinoamericanas. Desvincula la mente del cuerpo y a estas del espíritu.

El cuerpo humano es una maquina controlada por el corazón; el cerebro, su fundamento organizativo y su funcionalidad es dictada por la genética. Reduce el ser humano a órganos, células y moléculas; a términos de física, química y matemática; a la acción de enzimas y de hormonas.

Si aparece el desequilibrio, se busca la causa física con la prescripción subsiguiente de fármacos para curar. Sin embargo, ¿̇a cuántas personas efectivamente cura la Medicina con sus medicamentos, quimioterapia y radioterapia? Este esquema newtoniano materia, causalidad, anomalía es documentado por Roa (1997).

La física cuántica revindica la importancia vital del espíritu, de la mente en el proceso salud - enfermedad y de los campos de energía, postura holística que aún no ha logrado traspasar totalmente la puerta del vetusto sistema educativo. Reviso su inclusión a partir de la literatura académica circulando por Internet.

\section{Crisis del modelo alopático}

El diagnóstico tecnologizado y el tratamiento farmacológico de enfermedades es el fundamento de la educación médica y de la investigación. Estas responden a estructuras económicas y legales que buscan sostener al actual sistema de salud.

La enfermedad es concebida como entidad clínica distintiva: tiene su propia fisiopatología, historia natural, tratamiento aceptado y un código en la Clasificación Internacional de Enfermedades.

La formación académica de Enfermería ha sido forzosamente destinada a sostener este modelo, junto con la farmacéutica, adecuando su ejercicio al puesto de trabajo esperado por los sistemas, supeditando durante mucho tiempo su verdadero potencial.

Menéndez (1988) describe la Medicina en términos de “... biologismo, individualismo, ahistoricidad, asociabilidad, mercantilismo, eficacia pragmática, asimetría, autoritarismo, participación subordinada y pasiva del paciente, exclusión del conocimiento del consumidor, legitimación jurídica, profesionalización formalizada, identificación con la racionalidad científica, tendencias inductivas al consumo médico".

Históricamente, el modelo ha presentado submodelos vinculantes del proceso salud - enfermedad - atención médica (Arredondo, 1992; Madrid, 2011). Según León (2013), la Organización Mundial de la Salud (OMS, 1983), en su publicación titulada 
Medicinas tradicionales y la cobertura de la asistencia sanitaria, expresó que "la llamada medicina oficial no logra cumplir con sus objetivos de disminuir la morbimortalidad".

Los modelos de atención normalizan las prácticas sanitarias que, en Occidente y en Latinoamérica, siguen el esquema anglosajón con jerarquías disciplinarias simbolizando poder. Conocimientos y niveles de prevención se organizan verticalmente, desconsiderando las prácticas populares. Implican filosofías sobre persona, derechos, participación ciudadana, Deontología y relaciones con los profesionales del equipo para la salud.

Estos modelos rectores impregnan la epistemología de conceptos que subyacen en la teoría y en la práctica enfermera, a tal punto de haber imitado el método científico, transferido al clínico (Arteaga Herrera, 2010), al epidemiológico (Hernández Hernández, 2002) y al Proceso de atención de Enfermería (PAE) (Despaigne Pérez, 2015).

Así, la "doxa" no solo se contrapone a la natural "episteme" sino que la aparente racionalidad, menospreció ciertos saberes clasificados de esotéricos, religiosos, mitológicos y de brujas, algunos de ellos, hoy probados por las CC.

Las enfermeras, marcadas por el método científico, actúan con desconocimiento sobre sus limitaciones, concepciones epistemológicas, intereses ideológicos que lo sustentan y el choque ético con la posición hipocrática y maimonidista.

Puede decirse que un mejor cuidado enfermero está viendo la luz. Nuevas pruebas científicas reafirman la visión integrativa, incorporándola en los PAE.

Enfermería habría podido iniciar tempranamente su aplicación, si las condiciones sociales le habrán permitido seguir fielmente a sus teóricas y actuar con independencia clínica.

\section{Ciencias de la complejidad}

Vida y salud no cumplen con la linealidad, jerarquización y burocratización de la estructura médica, inspiración administrativa para Enfermería. El cuidado de la salud tiene múltiples facetas. Su estudio debe abordarse desde diferentes ángulos, vinculando entidades heterogéneas.

En plena "revolución científica" (Kuhn, 2004), es deseable que emerja una reorganización de estructuras, haceres y saberes, tendiendo a la eficacia con ahorro burocrático.

Basadas en la física cuántica, las matemáticas no lineales y la informática, buscan transdisciplinariedad con transferencia de conocimientos. Mayntz (2002) critica la clásica división entre ciencias duras y blandas: "las disciplinas científicas por más que pretendan configurarse como unidades grupales limpiamente delimitadas, difícilmente logran sistemas cerrados desde un punto de vista cognitivo. Al contrario, la historia evolutiva de la ciencia está plagada de cruces transfronterizos y de enriquecimientos recíprocos".

Científicamente, la Enfermería es interdisciplina (RÉ, 2018), concibiendo holísticamente el hombre, el entorno y el cuidado. Teorías y modelos de cuidado 
confluyen en el argumento de la Filosofía perenne. Los seres humanos estamos constituidos por la llamada Gran Cadena del Ser: somos materia, cuerpo, mente, alma y espíritu (Wilber, 1983). Es natural que incorpore los hallazgos de la física cuántica, entre otros, la que precisa que la materia es energía, superando y desvinculándose de las imposiciones de la ciega Medicina convencional.

Cuidar es la pieza clave ante el proceso salud - enfermedad para no llegar a esta condición que significa pérdida de energía. Por otro lado, "las curaciones espontáneas, los fenómenos psíquicos, las asombrosas demostraciones de fuerza y resistencia, la habilidad para caminar sobre las brasas de un fuego sin quemarse, la capacidad de la acupuntura para disminuir el dolor mediante la canalización del chi a lo largo del cuerpo y otros muchos fenómenos paranormales desafían la biología newtoniana" (Rico Becerra, 2010).

\section{Medicina cuántica}

La física cuántica indaga aspectos insondables desde la óptica del mundo físico de Newton, por ejemplo, el poder del pensamiento sobre el cuerpo y su salud, dando origen a la Medicina cuántica (Med. Qn), integrativa o transdisciplinaria.

Con lógica einsteiniana, utiliza el concepto de unidad cuántica para describir propiedades dinámicas de partículas subatómicas y las interacciones entre la materia y radiación, adentrándose al mundo de lo invisible y de la energía.

Ontológicamente, tiene en cuenta el comportamiento y propiedades de estas entidades subatómicas y sus formas causales de establecer relaciones que no obedecen a la rigidez. Sus supuestos son la incertidumbre, la no-identidad y la falta de localidad puntual, implicando emergencia de un nuevo tipo de cientificidad (López Mosqueda, 2017).

La Med. Qn considera la relación que cada persona tiene con su entorno, ya que todo en el universo está relacionado a una red energética vibratoria a través de paquetes de energía -denominados Quantum- y que actúan en una resonancia llamada coherencia.

Esta explica "profundamente con el sentido de comenzar a comprender cómo una desarmonía en nuestro ser, que en ocasiones se manifiesta como enfermedad, ya no es un mero " accidente " o " casualidad" (Nogués, 2001). La conciencia, los pensamientos y los sentimientos conscientes e inconscientes regulan la actividad mental, la emocional y la física, sin olvidar las vibraciones que nos entornan, aportando o disminuyendo la vitalidad.

La pérdida de coherencia o de entropía-término de la termodinámica, indica el grado de desorden molecular-hace surgir la enfermedad en un tejido, órgano o sistema. Un suministro de energía sutil o quantum restablece la armonía: medicamento homeopático, esencia floral, gemas, cristales, cromoterapia, acupuntura, masoterapia y toda terapia que implique transferencia de campos electromagnéticos, en calidad de vibraciones con efecto directo sobre el organismo. Instauran un nuevo orden en la persona, a partir de una reorientación manifestada a nivel celular, molecular y atómico. 
La OMS (2013) reconoció que la llamada Medicina Tradicional o Complementaria (MTC/MAC) "es una parte importante y con frecuencia subestimada de la atención de salud" y que existe "actualmente la necesidad de elaborar un enfoque coherente e integral de la atención de salud, que facilite a los gobiernos, los profesionales sanitarios y, muy especialmente, a los usuarios de los servicios de salud, el acceso a la MTC de manera segura, respetuosa, asequible y efectiva". La Medicina Integrativa es utilizada en un amplio espectro de enfermedades, especialmente las crónicas.

\section{Repercusiones políticas}

La estrategia OMS 2002-2005 es retomada para el período 2014-2023 instando a:1) la integración de la MT y la MAC;2) promover la seguridad, eficacia y calidad; 3) aumentar la disponibilidad y asequibilidad con énfasis en las poblaciones más pobres, y 4) fomentar el uso terapéutico sólido por parte de consumidores y proveedores.

Los gobiernos conscientes de sus beneficios trabajan para integrarlas en los servicios de salud (Caminal, 2006). El Parlamento Latinoamericano aprobó la Ley Marco en Materia de MAC para América Latina y el Caribe, regulando la práctica, enseñanza e investigación (PL, 2009).

Recomienda incorporar a los Sistemas Nacionales de Salud la acupuntura, la homeopatía, la quiropráctica y la herbolaria medicinal. El principio subyacente de la complementariedad permite diversas alternativas, destacando la conexión interactiva emoción y cognición (Swartz, 2003). Según el National Center For Complementary and Alternative Medicine (NCCAM), las terapias se agrupan en 5 categorías:

1- Medicina homeopática y naturópata, la medicina tradicional china y ayurveda.

2- Intervenciones cuerpo-mente: meditación, musicoterapia, danzaterapia, cromoterapia, aromaterapia etc.

3- Biológicas (se encuentran en la naturaleza): herboristería, fitoterapia, dietética, complementos alimenticios y alimentos funcionales.

4- Manipulación del cuerpo: quiropráctica y osteopatía, etc.

5- Terapias energéticas: Qi gong/Qigong o Chi Kung, Reiki, campos electromagnéticos, entre otras (González, E. y Quindós, 2010).

Con las CC "la administración médica se analiza interdisciplinariamente" considerando con seriedad la convergencia de factores éticos, sociales, materiales, económicos y humanos (Fajardo-Ortiz, 2013) agregándose los aportes de la MAC.

Sus ventajas son: humanización en el trato terapéutico, disminución de costos en medicamentos y en uso de servicios de alta complejidad (Pinto-Barrero, 2012), mínimos efectos adversos, aplicación significativa para el público y fácil acceso (Peña Martínez, 2018).

Existen experiencias médicas de complementariedad alopática-homeopática (Montes de Oca-Rosas, 2005). En Enfermería se emparentan con filosofías orientales, aplicadas al cuidado individual en diversas situaciones del amplio espectro salud- 
enfermedad. Son numerosas en territorio europeo, en contraposición con el latinoamericano. Son aplicadas en diversas especialidades y en distintos niveles de atención de la salud (Ahogado, 2008; Snyder, 2010; AEEU, 2012; Ceolin, 2009; GómezMartínez, 2018).

Su utilización en APS para preservarla es notoria. Enfermería es un recurso humano decisivo para el ahorro del gasto público sanitario y para brindar un servicio humanizado por el valor que otorga a la vida humana (Tobos de Álvarez, 2000; Gella-Jarne, 2014; Timis, 2017). La salud encarada con visión integral incorpora el concepto bienestar, distanciando la perspectiva veterinaria sobre cuerpos humanos, con conciencia de la dimensión espiritual, reflejado en el PAE (Facione, 2017; Sánchez Herrera, 2004, Pérez-García, 2016).

\section{Repercusiones disciplinarias}

La American Nurses Association (ANA) reconoció la Enfermería Holística como especialidad en noviembre 2006. En 2007, la ANA publicó un libro junto con la American Holistic Nursees Association (AHNA) normatizando objetivos, práctica, implementación en servicios y evaluación (AHNA y ANA, 2015). Aporta información a las enfermeras holísticas, otros profesionales sanitarios, empleadores, terceras partes financiadoras, legisladores y al público respecto de la visión única del conocimiento, de los estándares de práctica y de desempeño esperado en la enfermera holística.

Enfermería, fiel a la tradición hipocrática, insiste en la relación cuerpo, alma, ambiente, tal que las medicinas orientales. Desde hace 25 siglos, las Medicinas China, Tibetana y Ayurvédica, previenen y curan por el abordaje integral de la persona. Utilizan un procedimiento diagnóstico exhaustivo y una intervención terapéutica personalizada, que incluye indicaciones dirigidas a modificar los estilos de vida.

Reconocen las capacidades preventivas autorreparadoras endógenas del ser humano, como la estimulación de la oxitocina, justificado en el concepto naturae medicatrix (la naturaleza es la que cura), conservado en los médicos naturistas. Estas filosofías consideran las capacidades sanadoras innatas del organismo.

La espiritualidad es "la disciplina que enmarca: la filosofía, la religión y la ciencia y trata de armonizarla" (op.cit, Montes de Oca-Rosas, 2005). Pinedo Velázquez (2009) inquirió los beneficios de las romerías ilicitanas en personas enfermas con el propósito de "reconocer el peso que para un determinado paciente tienen sus creencias, cuantificando de algún modo la vivencia del bienestar que le producen y relacionar esas emociones con su estado de salud" porque "la fe tiene un efecto energético en las personas creyentes que les infunde fuerza para luchar contra un problema de salud".

La salud fractal incluye esta dimensión (Guirao Goris, 2013) y el corpus literario académico demuestra un creciente interés por la religiosidad, la espiritualidad y su incidencia preservativa (Valiente-Barroso, 2010; Salgado, 2014; Morales-Ramón, 2014; Ureña Bonilla, 2014). La salud se concibe con las nociones de autoestima, optimismo, felicidad, bienestar y desarrollo personal. 


\section{Repercusiones académicas}

La formación profesional es holística desde sus raíces emparentadas con la Ecofeminismo. "En este sentido, las teorías que se han creado y divulgado en la ciencia de Enfermería han basado sus principios, conceptos y definiciones en un modelo integral, multidimensional y complejo, que se deriva de un enfoque holístico del cuidado" (Mijangos-Fuentes, 2014). El holismo destaca en las filosofías de Martha Rogers, Jean Watson, Betty Neuman, Paterson y Zderad (Chalco Castillo, 2019; Meleis, 2012).

El integrismo contempla la dimensión espiritual con los Principios de bioenergética o Termodinámica. En general, "las enfermeras como grupo de profesionales de la salud son las que más solicitan preparación en esta área, su cercanía con los pacientes les permite aplicar con mayor facilidad procedimientos complementarios autorizados $y$ vigilados por el personal médico" (Dossey M, 1998).

La Clasificación de Intervenciones de Enfermería (NIC) incluye las MAC (Bulechek, 2014). Ciertas universidades las dispensan como parte de la malla curricular de Medicina y de Enfermería (Valarezo-García, 2017; Cáceres Guido, 2009; López Ruiz, 2009). Numerosísimos son los ejemplos de investigaciones efectuadas en la temática (Ballesteros-Peña,2015; Ceballos Vásquez, 2016; Calderón Avellaneda, 2017; Galán, 2019; Ruiz Gómez, 2005; Ávila-Sansores, 2010). Incluidas en espacios académicos de formación de grado y posgrado, influyen directamente en la intervención a posteriori (Esc. De Enfermería Dr. Darcio Crespo, 2018; Gutiérrez, 2007; Pecina Leva, 2011; Universidad Católica de Murcia, 2018 y de Burgos, 2018).

\section{Conclusiones}

“De la misma manera en que concluimos que no podíamos confiar las metas de la defensa nacional al complejo militar-industrial, estamos empezando a entender la inconveniencia de encomendar la medicina científica al complejo médico-industrial." $E$. Golub

“Enfermería ha perdido su alma por servir a la medicina, la tecnología y la práctica basada en la evidencia, las que por cierto se necesitan; pero sin la dimensión humana, esto puede ser destructivo. Es fundamental preservar la esencia humana y reposicionar el cuidado dentro de nuestros sistemas" Jean Watson (Urra, 2011).

El reduccionismo pedagógico impuesto por el biologicismo médico queda superado por el avance y la invitación de las CC a salir de la programación lineal y material, que persisten en el sistema educativo académico.

Esta revisión dispara la discusión sobre las representaciones, el valor, los conocimientos, las aptitudes y las actitudes asignadas a las MAC en el currículum enfermero, en docentes, alumnos y enfermeros en ejercicio. Confirmó su integración universitaria y en la atención de servicios de salud (ámbito público y privado) en distintos contextos socioculturales. 
Su efectiva introducción en la formación de grado y posgrado permitiría brindar servicios con seguridad, regulados legalmente, facilitando la docencia, la investigación y la transferencia, mientras se protege al público.

Las MAC se ubican perfectamente en el modelo APS definido por OMS en 1978 en la Declaración de Alma-Ata como "la asistencia sanitaria esencial puesta al alcance de todos los individuos y familias de una comunidad, por medios que le sean aceptables, con su plena participación y a un coste que la comunidad y el país puedan soportar", las que son bien aceptadas por la ciudadanía mundial (2012).

Las inversiones económicas para proveer la salud se deben concentrar en la APS. Es inteligente mermar recursos en áreas que presentan menores rendimientos con tecnología costosa. El modelo epidemiológico llega tarde en la vida de la persona porque ésta ya está enferma. No se adapta a las necesidades de la población que quiere mantenerse con bienestar.

Las CC y la MAC son coherentes con la Filosofía, el corpus intelectual, los valores y las virtudes de las enfermeras y ellas deben estar presentes en los PAE, consiguiendo cuidados y cuidadores holísticos, respetuosos de los derechos de los pacientes. Recordemos lo expresado por Virginia Henderson: "si las enfermeras no hacen lo que deben hacer, personas menos preparadas lo realizaran".

\section{Referencias bibliográficas}

AHOGADO, C. (2008). Experiencias de profesionales de enfermería en terapias alternativas y complementarias aplicadas a personas en situaciones de dolor. Revista Avances de la Enfermería, Volumen 26, Número 1, p. 59-64. Disponible en https://revistas.unal.edu.co/index.php/avenferm/article/view/12885/13643

AMERICAN NURSES ASSOCIATION. (2015). Nursing: Scope and Standards of Practice. Third Edition. Published by Nursesbooks.org. The Publishing Program of ANA. Disponible en https://www.iupuc.edu/health-sciences/files/Nursing-ScopeStandards-3E.pdf

ARREDONDO, A. (1992). Análisis y Reflexión sobre Modelos Teóricos del Proceso Salud Enfermedad. Disponible en http://www.scielo.br/pdf/csp/v8n3/v8n3a05

ARTEAGA HERRERA, J. (2010). El método clínico y el método científico. MediSur, vol. 8, núm. 5, pp. 12-20. Universidad de Ciencias Médicas de Cienfuegos, Cienfuegos, Cuba. Disponible en https://www.redalyc.org/pdf/1800/180020098003.pdf

ASOCIACIÓN ESPAÑOLA DE ENFERMERÍA EN UROLOGÍA (AEEU). (2012). Las terapias naturales en la enfermería. Aplicación en los procesos urológicos. ENFURO 38. Núm., 122. Mayo/Junio/Julio/Agosto. Disponible en https://dialnet.unirioja.es/descarga/articulo/4274042.pdf

ÁVILA-SANSORES, G. M. (2010). Efecto del Reiki como cuidado de enfermería en el control metabólico de diabéticos tipo 2. Rev Enferm Inst Mex Seguro Soc 2010; 18 (2): 75 - 
80. Disponible en https://www.medigraphic.com/pdfs/enfermeriaimss/eim2010/eim102c.pdf

BALLESTEROS-PEÑA, S. (2015). Conocimientos y actitudes sobre terapias alternativas y complementarias en estudiantes de ciencias de la salud. Revista de Educación Médica. Disponible en http://riem.facmed.unam.mx/node/444

BULECHEK, G. (2014). Clasificación de Intervenciones de Enfermería (NIC). Ed. Elsevier. 6ta edición.

Disponible

en

https://www.academia.edu/37376104/CLASIFICACION_DE_INTERVENCIONES_DE_E NFERMERIA_NIC

CÁCERES GUIDO, P. (2009). Medicinas complementarias, alternativas e integradora: encuesta sobre conocimiento, uso y opinión en el personal de enfermería de un hospital pediátrico de alta complejidad. Disponible en http://www.medicinainfantil.org.ar/images/stories/volumen/2009/xvi_1_024.pdf

CALDERÓN AVELLANEDA, E. (2017). Nivel de conocimiento sobre medicina alternativa sobre medicina alternativa en los docentes de la Facultad de Ciencias de la Salud de la Universidad Nacional Toribio Rodriguez de Mendoza de Amazonas, Disponible en http://docs.bvsalud.org/biblioref/2018/11/948866/nivel-de-conocimiento-sobremedicina-alternativa-en-los-docente_QG9V0yR.pdf

CAMINAL, J.; RODRÍGUEZ, N. (2006). Las medicinas complementarias y alternativas y su contribución al sistema sociosanitario: el qué, el porqué y el cómo. Elsevier, Revista Atención Primaria. Vol. 38. Núm. 7. Páginas 409-412. Disponible en https://www.elsevier.es/es-revista-atencion-primaria-27-articulo-las-medicinascomplementarias-alternativas-su-13094059

CEBALLOS VÁSQUEZ, P. A. (2016). Propuesta de enfermería con terapias complementarias para el abordaje de riesgos psicosociales a nivel laboral. Revista Enfermería Actual, Costa Rica. Disponible en https://www.scielo.sa.cr/pdf/enfermeria/n30/1409-4568enfermeria-30-00108.pdf

CEOLIN, T. (2009). Inserción de terapias complementarias en el sistema único de salud atendiendo al cuidado integral en la asistencia. Disponible en http://scielo.isciii.es/scielo.php?script=sci_arttext\&pid=S1695-61412009000200017

CHALCO CASTILLO, N. (2019). Epistemología en Enfermería. Universidad Nacional del Callao Disponible

en http://repositorio.unac.edu.pe/bitstream/handle/UNAC/4187/Chalco\%20Castillo_IF _2019.pdf?sequence=1\&isAllowed=y

DESPAIGNE PÉREZ, C. (2015). El Proceso de Atención de Enfermería como método científico. Revista 16 de abril. Cuba. Páginas 91-96. Disponible en https://www.medigraphic.com/pdfs/abril/abr-2015/abr15259j.pdf

DOSSEY, M.; DOSSEY, L. (1998). Body-Mind-Spirit. American Journal of Nursing, 1998; 98 : 35-8. Citado por Montes de Oca-Rosas, D. Disponible en https://www.medigraphic.com/pdfs/sanmil/sm-2005/sm056i.pdf 
ESC. DE ENFERMERÍA DR. DARCIO CRESPO. (2018). Intervención de Enfermería en Terapias Alternativas. Disponible en https://www.diputaciondepalencia.es/system/files/generico/archivos/20180411/at encion_de_enfermeria_en_terapias_alternativas.pdf

FACIONE, P. A. (2017). Pensamiento Crítico Holístico en el Proceso Diagnóstico de Enfermería. [Editorial]. Rev Gaúcha Enferm. 2017; 38(3): e75576. doi: http://dx.doi.org/10.1590/1983-1447.2017.03.75576. Disponible en https://www.scielo.br/pdf/rgenf/v38n3/es_0102-6933-rgenf-38-3-e75576.pdf

FAJARDO-ORTIZ, G. (2013). El enfoque de las ciencias de la complejidad en la administración de servicios de salud. Rev Med Inst Mex Seguro Soc. 2013; 51 (2):164-9. Disponible en https://www.medigraphic.com/pdfs/imss/im2013/im132i.pdf

GALÁN, A. N. (2019). El camino hacia la humanización de los cuidados. Cuidados al paciente Adulto Hospitalizado. Universidad de Alicante. Disponible en https://rua.ua.es/dspace/bitstream/10045/92831/1/El_camino_hacia_la_humaniza cion_de_los_cuidados_Nieves_Galan_Andrea.pdf

GELLA-JARNE, H. (2014). Las terapias naturales, un instrumento de cuidados. Revista Medicina Naturista, 2015; Vol. 9 - № 1: 25-30. Disponible en https://dialnet.unirioja.es/descarga/articulo/4952946.pdf

GÓMEZ-MARTíNEZ, R. (2018). Usos y actitudes del personal de enfermería acerca de las terapias alternativas en un hospital pediátrico. Rev Enferm Inst Mex Seguro Soc. 2018;26(2):115-20.

Disponible

en

https://www.medigraphic.com/pdfs/enfermeriaimss/eim-2018/eim182h.pdf

GONZÁLEZ, E. y QUINDÓS, I. (2010). La incorporación de terapias naturales en los servicios de salud. Escuela universitaria de Enfermería Santa Madrona. Disponible en http://diposit.ub.edu/dspace/bitstream/2445/21702/1/2009_8.pdf

GUIRAO GORIS, J. A. (2013). La espiritualidad como dimensión de la concepción holística de salud. ENE. Revista de Enfermería. Abr. 2013; 7 (1). Disponible en http://eneenfermeria.org/ojs/index.php/ENE/article/download/252/206

GUTIÉRREZ, B. (2007). Terapias complementarias en la Escuela de Enfermería. Universidad de Jaén. Revista Enfermería Global Numero 10, paginas 1-8. Disponible en http://revistas.um.es/eglobal/article/viewFile/254/210

HERNÁNDEZ HERNÁNDEZ, R. (2002). Del método científico al clínico. Consideraciones teóricas. Filial de Ciencias Médicas de la Isla de la Juventud, municipio Especial Isla de la Juventud, Cubar Disponible en http://scielo.sld.cu/scielo.php?script=sci_arttext\&pid=s0864-21252002000200011 KUHN, T. (2004). La estructura de las revoluciones científicas. Fondo de Cultura Económico, Buenos Aires. Disponible en https://materiainvestigacion.files.wordpress.com/2016/05/kuhn1971.pdf

LEÓN, C. (2013). Enfermería y terapias alternativas. Disponible en https://es.slideshare.net/mayrileon/enfermera-y-terapias-alternativas 
LÓPEZ MOSQUEDA, J. A. (2017). La filosofía frente al objeto cuántico. Revista Mexicana de

Física. E 63 (2017) paginas 107-122. Disponible en http://www.scielo.org.mx/pdf/rmfe/v63n2/1870-3542-rmfe-63-02-107.pdf

LÓPEZ RUIZ, J. (2009). Bases para a acreditación de la competencia de los profesionales enfermeros en terapias naturales y complementarias. Disponible en https://pbcoib.blob.core.windows.net/coib-publish/invar/4536394c-abfd-41a1b5bd-3bca73bd8403

MADRID, L. B. (2011). Realizando 'diagnósticos diferenciales' de los modelos teóricos del proceso salud-enfermedad. Disponible en https://www.revistakairos.org/realizandodiagnosticos-diferenciales-de-los-modelos-teoricos-del-proceso-salud-enfermedad/

MAYNTZ, R. (2002). Modelos científicos, teoría sociológica y el problema macro-micro. Revista Española de Investigaciones Sociológicas (REIS). No. 98 (abril - junio, 2002), págs. 65-78. Disponible en http://www.jstor.org/discover/10.2307/40184438?uid=3738664\&uid=2129\&uid=2\& uid $=70 \&$ uid $=4 \&$ sid $=21101570435793$

MELEIS, A. (2012). Theorical nursing. Development and Progress. 5 th edition. Disponible en

https://www.academia.edu/30976329/Theoretical_Nursing_Development_and_Pro gresss.pd1f

MENÉNDEZ, E. L. (1988). Modelo Médico Hegemónico y Atención Primaria. Segundas Jornadas de Atención Primaria de la Salud. 198830 de abril al 7 de mayo. Buenos Aires. 1988 Pág. 451-464. Disponible en https://www.psi.uba.ar/academica/carrerasdegrado/psicologia/sitios_catedras/elec tivas/816_rol_psicologo/material/unidad2/obligatoria/modelo_medico.pdf

MIJANGOS-FUENTES, K. I. (2014). El Paradigma Holístico de la Enfermería. Salud y Administración Volumen 1 Número 2 Mayo-Agosto. Disponible en http://www.unsis.edu.mx/revista/doc/vol1num2/A3_Paradigma_Holistico.pdf.

MONTES DE OCA-ROSAS, D. (2005). La medicina alternativa y complementaria, una opción institucional. Instituto Mexicano del Seguro Social. Academia Mexicana de Cirugía. Ciudad de México. Disponible en https://www.medigraphic.com/pdfs/sanmil/sm2005/sm056i.pdf

MORALES-RAMÓN, F.; Ojeda-Vargas, M. G. (2014). El cuidado espiritual como una oportunidad de cuidado y trascendencia en la atención de enfermería. Revista Salud en Tabasco, Vol. 20, No. 3, Septiembre-Diciembre 2014. Disponible en https://tabasco.gob.mx/sites/default/files/users/ssaludtabasco/94.pdf

NOGUÉS, I. (2001). Física cuántica y conciencia. Revista Natura Medicatrix, año 2001, Vol. 19, Número 5, páginas: 222-227. Disponible en https://dialnet.unirioja.es/descarga/articulo/4990799.pdf.

OMS. (2012). Conferencia Internacional sobre Atención Primaria de Salud, Alma-Ata, URSS, 6-12 de septiembre de 1978. Disponible en https://www.paho.org/hq/dmdocuments/2012/Alma-Ata-1978Declaracion.pdf 
OMS. (2013). Estrategia de la OMS sobre medicina tradicional 2014 - 2023. Disponible en https://apps.who.int/iris/bitstream/handle/10665/95008/9789243506098_spa.pdf;j sessionid=AF52F02B51C7CC22E48B3B492F20B0EF?sequence $=1$

PARLAMENTO LATINOAMERICANO. (2009). XXV Asamblea ordinaria parlamento latinoamericano. Disponible en http://parlatino.org/pdf/organosprincipales/asamblea/actas/xxv_asamblea-pma-3-12-2009.pdf

PECINA LEVA, R. M. (2011). El proceso enfermero desde la perspectiva holística del Healing touch. Enf Neurol (Mex) Vol. 10, No. 2: 68-71, 2011. Disponible en https://www.medigraphic.com/pdfs/enfneu/ene-2011/ene112c.pdf

PEÑA MARTÍNEZ, A. C. (2018). Integración de las terapias alternativas y complementarias en el Sistema Nacional de Salud. Universidad Autónoma de Madrid. Disponible en https://repositorio.uam.es/bitstream/handle/10486/685022/pena_martinez_ana\%2 Ocristinatfg.pdf?sequence $=1$ \&isAllowed $=y$

PÉREZ-GARCÍA, E. (2016). Enfermería y las necesidades espirituales en el paciente con enfermedad en etapa terminal. Revista Enfermería: Cuidados Humanizados, vol.5 no.2 Montevideo dic. Disponible en http://www.scielo.edu.uy/scielo.php?script=sci_arttext\&pid=\$239366062016000200006

PINEDO VELÁZQUEZ, M. T. (2009). Significado de las romerías populares y de las prácticas simbólicas en torno al proceso salud enfermedad en Elche: una aportación desde la perspectiva fenomenológica. Universidad de Alicante. Disponible en https://rua.ua.es/dspace/bitstream/10045/13431/1/tesis_pinedo.pdf

PINTO-BARRERO, M. (2012). Integración de la medicina alternativa en los servicios de salud de Colombia. Universidad La Sabana. Disponible en https://aquichan.unisabana.edu.co/index.php/aquichan/article/view/2032/2930

RÉ, C. (2018). La enfermería, una ciencia interdisciplinaria. Revista ROL de enfermería, Vol. 41, № 3, 2018, págs. 38-49.

RICO BECERRA, J. I. (2010). Enfermería, cuidado y ciencia. Revista Cultura de los Cuidados. 2 ‥ Semestre 2010 • Año XIV - N. ${ }^{\circ} 28$, pps. 76-82. Disponible en http://www.indexf.com/cultura/28pdf/28-076.pdf

ROA, R. (1997). Proceso Salud y Enfermedad: Crisis del Paradigma Biomédico. Atención Primaria y Medicina Familiar. Tomo 1. 1. Buenos Aires Akadia Editorial. Disponible en

https://www.smu.org.uy/crep/material/saludyenfermedad_paradigmasycrisis.pdf

RUIZ GÓMEZ, M. C. (2005). Terapias complementarias en los cuidados. Humor y risoterapia. Revista Index de Enfermería, vol.14 no.48-49, Granada. Disponible en http://scielo.isciii.es/scielo.php?script=sci_arttext\&pid=s1132-12962005000100008

SALGADO, A. C. (2014). Revisión de estudios empíricos sobre el impacto de la religión, religiosidad y espiritualidad como factores protectores. Ene.-Jun. 2014, Vol. 2, No 1 : pp.121-159. Disponible en https://dialnet.unirioja.es/descarga/articulo/5475193.pdf 
SÁNCHEZ HERRERA, B. (2004). Dimensión espiritual del cuidado de Enfermería en situaciones de cronicidad y muerte. Bogotá: Universidad Nacional de Colombia. Facultad de Enfermería. Disponible en https://core.ac.uk/download/pdf/11052362.pdf

SCHWARTZ, G. E. (1991). The Data Are Always Friendly: A Systems Approach to Psychotherapy Integration. Journal of Psychotherapy Integration. 1:55-70. Citado por Schwartz, Gary E.; Russek, Linda G. (2003). El desafío de una medicina: Teorías de la Salud y ocho. Hipótesis del Mundo. Polis, Revista de la Universidad Bolivariana, vol. 1, núm. 5, invierno. Universidad de Los Lagos. Santiago, Chile. Disponible en https://www.redalyc.org/pdf/305/30500518.pdf

SNYDER, M. (2010). Terapias complementarias y alternativas en enfermería. Editorial Manual Moderno. Disponible en https://zoe.gnomio.com/pluginfile.php/3271/mod_resource/content/2/Terapias\%2 0Complementarias\%20y\%20Alternativas\%20en\%20Enfermeria.pdf

TIMIS, M. Á. (2017). Las Terapias Complementarias y el pensamiento enfermero, una nueva visión del cuidado. Escuela de Enfermería de Zamora. Disponible en https://gredos.usal.es/bitstream/handle/10366/135744/TG_TimisM.pdf;jsessionid= 78290876FA6243A558A0C7733BDE01BB?sequence $=1$

TOBOS DE ÁLVAREZ, L. S. (2000). Promoción de la vida saludable complementado con terapia alternativa. págs. 241-243. Disponible en http://biblio3.url.edu.gt/Publi/Libros/CyPdeEnfermeria/10.pdf.

UNIVERSIDAD CATÓLICA DE MURCIA. (2018). Terapias Complementarias. Grado en Enfermería. Guía Docente 2017/2018. Disponible en https://www.ucam.edu/sites/default/files/estudios/grados/gradoenfermeria/presencial/plan-de-estudios/17-18/curso-

3/62_2_38_opt_terapias_complementariasl_.pdf

UNIVERSIDAD DE BURGOS. (2018). Terapias Complementarias aplicadas a los Cuidados de Enfermería. Disponible en https://ubuvirtual.ubu.es/mod/guiadocente/get_guiadocente.php?asignatura=7219 \&cursoacademico $=2017$.

UREÑA BONILLA, P. (2014). Bienestar psicológico, espiritualidad en el trabajo y percepción subjetiva de la salud en personal académico y administrativo de la Universidad Nacional. Revista Electrónica Educare. Disponible en https://www.scielo.sa.cr/scielo.php?script=sci_arttext\&pid=S140942582014000100008

URRA, M. E. (2011). Algunos aspectos esenciales del pensamiento de Jean Watson y su teoría de cuidados transpersonales. Rev. Ciencia y Enfermería, vol.17 no.3 Concepción dic. Disponible en https://scielo.conicyt.cl/pdf/cienf/v17n3/art02.pdf

VALAREZO-GARCÍA, C. (2017). Integración de la medicina alternativa en la malla curricular de las carreras de Medicina y Enfermería de las universidades peruanas. Revista Educación Médica, Vol. 20. Núm. 2. páginas 118-124 (Marzo - Abril 2019). Disponible 
en https://www.elsevier.es/es-revista-educacion-medica-71-avance-resumenintegracion-medicina-alternativa-malla-curricular-1575181318300858

VALIENTE-BARROSO, C. (2010). La religiosidad como factor promotor de salud y bienestar para un modelo multidisciplinar de atención psicogeriátrica. Viguera Editores SL 2010. PSICOGERIATRÍA 2010; 2 (3): 153-165. Disponible en https://www.ucm.es/data/cont/docs/140-2013-10-04-documento25550.pdf WILBER, K. (1983). Gracia y coraje. En la vida y en la muerte de Treya Killam Wilber. Ed. Gaia. Madrid.

* Cecilia Ré: Dra. en Pensamiento Complejo, Educación. Multiversidad Mundo Real Edgar Morin (Hermosillo, Sonora, México). Mgt. En Educación, ort. Formación a Distancia (Téluq, UQAM, Québec, Canadá). Esp. En Docencia Universitaria (UTN, San Nicolás, Argentina.). Lic. En Enfermería (UNC, Córdoba, Arg.). Tec. Univ. En Com. Soc. (UNPA, Santa Cruz, Argentina). [E-mail: cre@uarg.unpa.edu.ar] 